\title{
SLAG SKIMMING JUDGMENT GAUGE DEVELOPMENT AT ARCELORMITTAL FLAT CARBON SOUTH AMERICA*
}

\author{
Clebson Joel Mendes de Oliveira \\ Roberto Dalmaso ${ }^{2}$ \\ Henrique Silva Furtado ${ }^{3}$
}

\begin{abstract}
Sulfur control in steel have been challenging process experts since the discovering of it deleterious effect on most of steel product properties. To separate this slag from hot metal is mandatory to complete desulfurization process. The preferred method is to skim hot metal surface physically removing the contaminated slag, attributing to the operator the evaluation of skimming efficiency. Thus, we present a new lower cost system using image processing methods foreseeing a future full automation skimming process.
\end{abstract}

Keywords: Image processing; Slag skimming; Steel making.

1 Computer Engineer/Master's Degree in Electrical Engineering, Automation Specialist, Process Automation Engineering, ArcelorMittal Tubarão, Vitória, Espírito Santo, Brasil.

2 Process and Automation Engineer/Master's Degree in Electrical Engineering, Automation Specialist, Process Automation Engineering, ArcelorMittal Tubarão, Vitória, Espírito Santo, Brasil.

3 Metallurgical Enginner/PhD Degree in Metallurgical Engineering, Steelmaking Specialist in Process Development, Steelmaking Plant Technical Staff, ArcelorMittal Tubarão, Vitória, Espírito Santo, Brasil. 


\section{INTRODUCTION}

Sulfur control in steel have been challenging process experts since the discovering of it deleterious effect on most of steel product properties. Despite the availability of modern steelmaking hot metal desulfurization, which is capable to achieve low sulfur content in hot metal, like 10ppm, it is hard to reach such sulfur level in steel without a costly extra steel desulfurization process. This is due to the fact that steelmaking processes involve oxidation; an environment that favors any sulfur from materials charged in the refining reactor contaminates back the metal.

Up to present date, sulfur has been removed from hot metal trough materials additions that generate a less dense slag phase rich in sulfur. Therefore, separating this slag from hot metal is mandatory to complete desulfurization process. The preferred method is to skim hot metal surface to physically remove the contaminated slag.

In most of hot metal desulfurization facilities, manually controlled skimming processes are the preferred choice, letting the evaluation of skimming efficiency at operator's hand. Despite the skill of operators, there is always a tradeoff: skimming too much means increase hot metal losses; skimming too less means too much sulfur contamination in steel.

Evaluation of skimming process is basically a visual task. Based on this concept one can find in market some high investment costs control system, which promises a better control. However, it is hard to evaluate the actual return of such an investment, basically due to the destination of hot metal losses and, also, due to steel composition mix.

The purpose of present work is to present a new lower cost system using image processing methods [1] foreseeing a future full automation skimming process.

\section{MATERIAL AND METHODS}

The goal of the proposed system is to estimate the volume of the slag continuously over all the skimming process. For this, it was proposed and developed an innovative pilot project, using image processing techniques.

An important premise assumed for this project was: to develop it in a short period of time and use only internal human resources. Therefore, the project was split in four main steps: system prototyping, code translation, implementation and testing.

Although the present state of the art in artificial vision is largely developed in many fields, working with these techniques is challenging, because of parameters definitions. For example, to segregate two well-defined classes in an image, is something easily performed by using thresholding algorithms. But, the threshold determination itself can be a difficult task.

Because of that, our approach to the project development was to start with a prototyping step using Matlab [2]. Matlab is a powerfull and flexible interactive set of tools for technical computing such as: numeric computation and visualization, linear algebra, statistics, filtering optimization, image processing, automatic control, etc. This approach is justified by productivity gains mainly in techniques choice and parameters definition.

After tests executions and completion in Matlab, using images from the real process, it was started to translate the generated codes to the final system programing language, Microsoft .Net C\#. This step has taken just 2 days because of Matlab's language similarities with $\mathrm{C \#}$. 
Once the initial steps (algorithms and parameters definition) were achieved, the final system implementation process was started, comprising: modeling, implementation, online tests and general validation. The full project development has taken no more than 3 months.

\subsection{Image Processing Approach}

In Matlab prototyping step, different image processing techniques, to distinguish slag from hot metal, were tested, including: RGB [3], near infrared (NIR) [4] and HSV [5] color space images. After some tests, it was concluded that the estimated volume index of the slag could be better calculated using NIR images.

The main task of the algorithm is to correlate slag volume with temperature gradient. The initial skimming process slag volume is used to normalize the calculated index in a standardized (percentage) range. Figure 1 presents an instantaneous 3D volume approximation and its respective volume index, calculated by the system prototype using Matlab.

The horizontal plane represents the image dimension in pixels, 1296x966, and the vertical axis its respective height of slag, a value inversely proportional to current pixel value. The volume index can be estimated by summing up all the heights over the image plane. Figure 1 shows one example where the estimated volume index of slag is equals to $6.1 \%$.

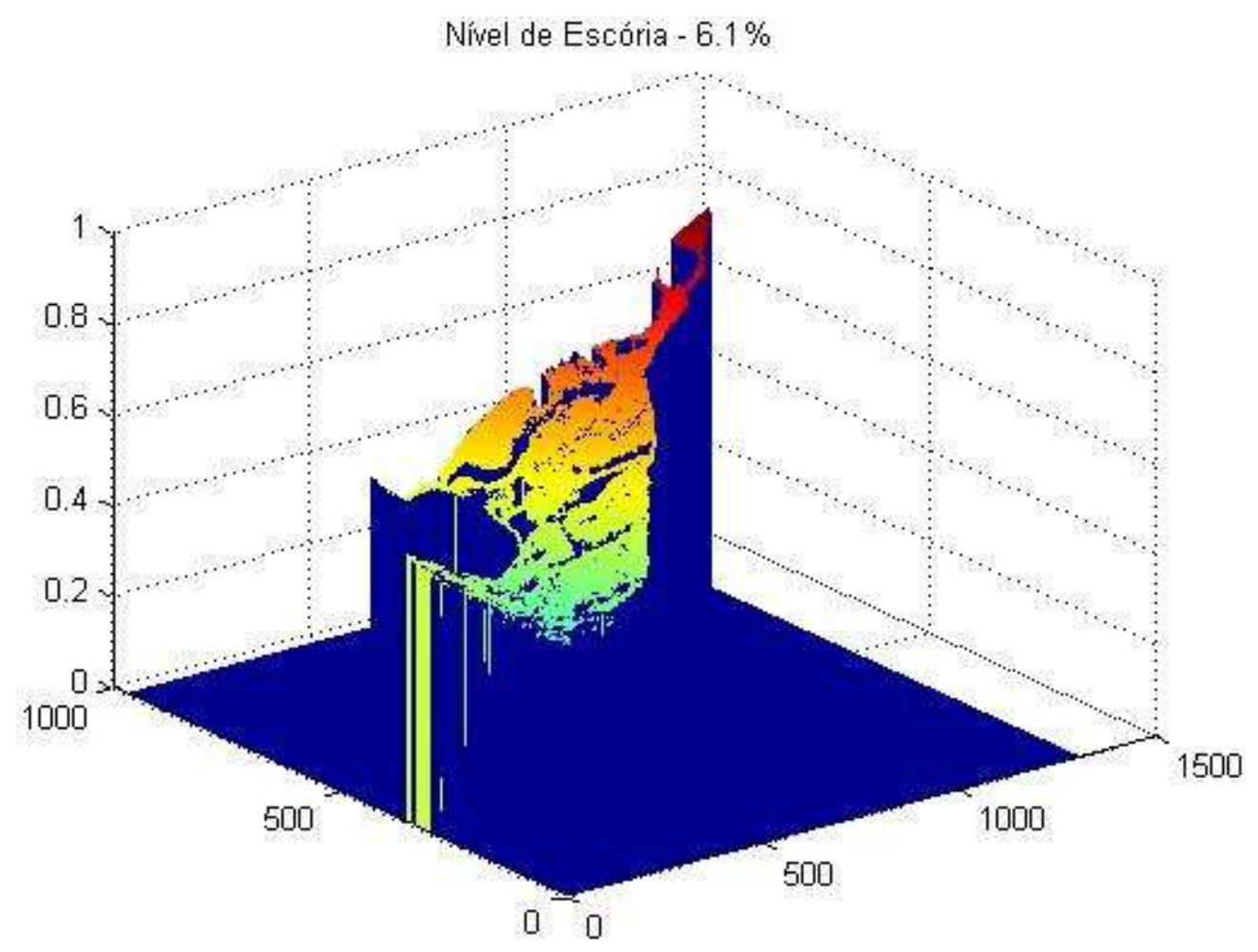

Figure 1. Slag 3D volume approximation using Matlab.

\section{RESULTS AND DISCUSSION}

This section presents an overview of the required infrastructure to run the system and the pilot system developed, covering all the hardware (cameras, network and computers) and software (applications and algorithms). 


\subsection{System Infrastructure}

The complete system comprises of three dual CCDs cameras, a Gigabit Ethernet switch and an industrial computer, as presented in Figure 2Erro! Fonte de referência não encontrada.. The industrial computer is responsible for running the gauge algorithms, and also, to communicate with the process computer (PROCOM) and the PLC responsibles for controlling the KR desulfurization process. The PROCOM sends the targeted sulfur value and the treatment id number to the gauge in order to optimize and control each desulfurization treatment. The cameras are installed on air blown refrigerated jackets, with dust protection, and far from the skimming process due to material projection and the emitted heat. The dual CCD cameras used (Figure 3) presents a color CCD (RGB) and a near infrared CCD (NIR), and the captured image is pixel by pixel aligned between them. The image acquisition is controlled by software as well as all the other parameters (exposure time and gain).

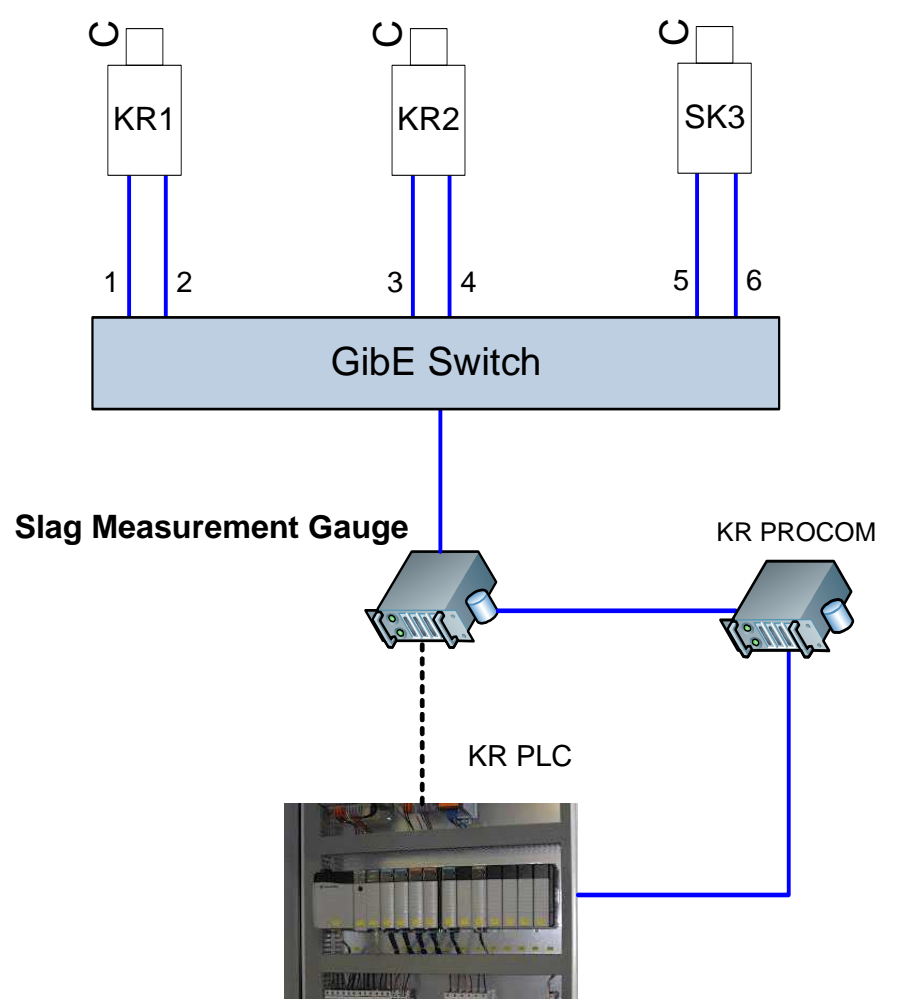

Figure 2. System architecture

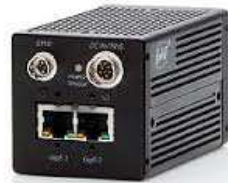

Figure 3. Dual CCD camera

\subsection{Project Costs}

The total invested capital in the project was low, especially when compared with similar industrial systems, and is estimated to reach, at the end of the project ( $2 \mathrm{KRs}$ +1 skimming installation), around 100kUSD, comprising the material acquisition 
(cameras, industrial computer and lenses) and the installation services (cabling, piping, environment and ergonomic maintenance issues).

\subsection{User Application}

One of the main requirement of the system is to be simple to use and easy to understand. A characteristic image acquired by the system is presented in Figure 4. The RGB colored image is shown on the left side, whilst, the right side shows the NIR image, or a slag image calculated using the NIR image, depending on the system state.

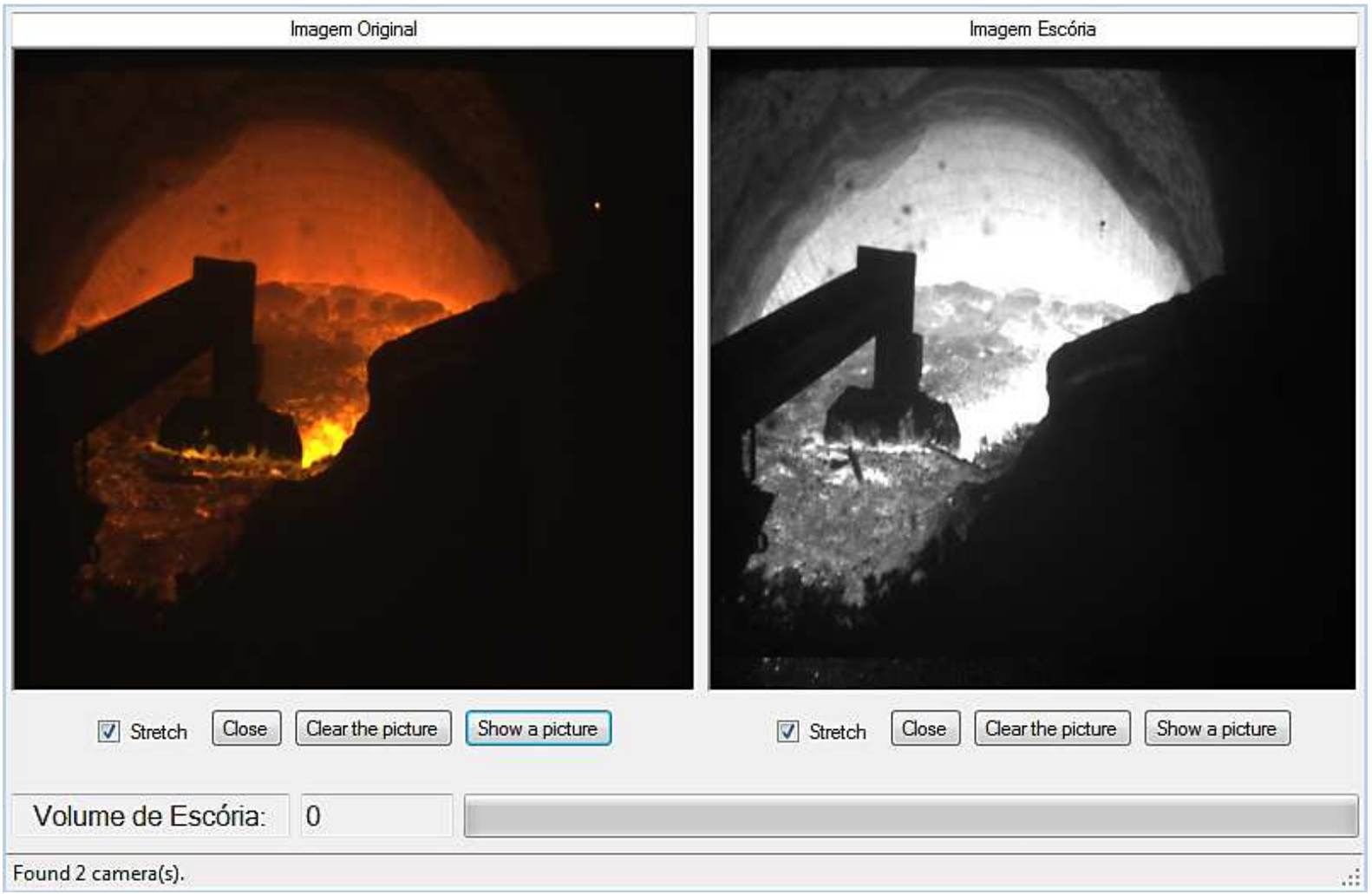

Figure 4. Screenshot from first pilot system at the beginning of a skimming process.

Figure 4 presents the first version of the pilot system, which tried to automatically calculate the initial slag volume, necessary to normalize the slag index. However, this has been proven not to be a good method, considering that, determining automatically the beginning of the skimming process can be a complicated issue. During the online tests, some situations where a delay on the skimming operation after the hot metal ladle arrival in front of the skimmer, were observed as a normal standard, and, conditions like these, may cause a decrease in performance evaluation because of the operational delay.

Thereby, the system has been modified to a new way of working, shown in Figure 5Figure 5. At present, operators have to manually inform the beginning of a new skimming process, and also, by finalizing the current one, using the buttons pointed by the red arrows. 


\section{9th Industrial $\Pi$ and Automation}

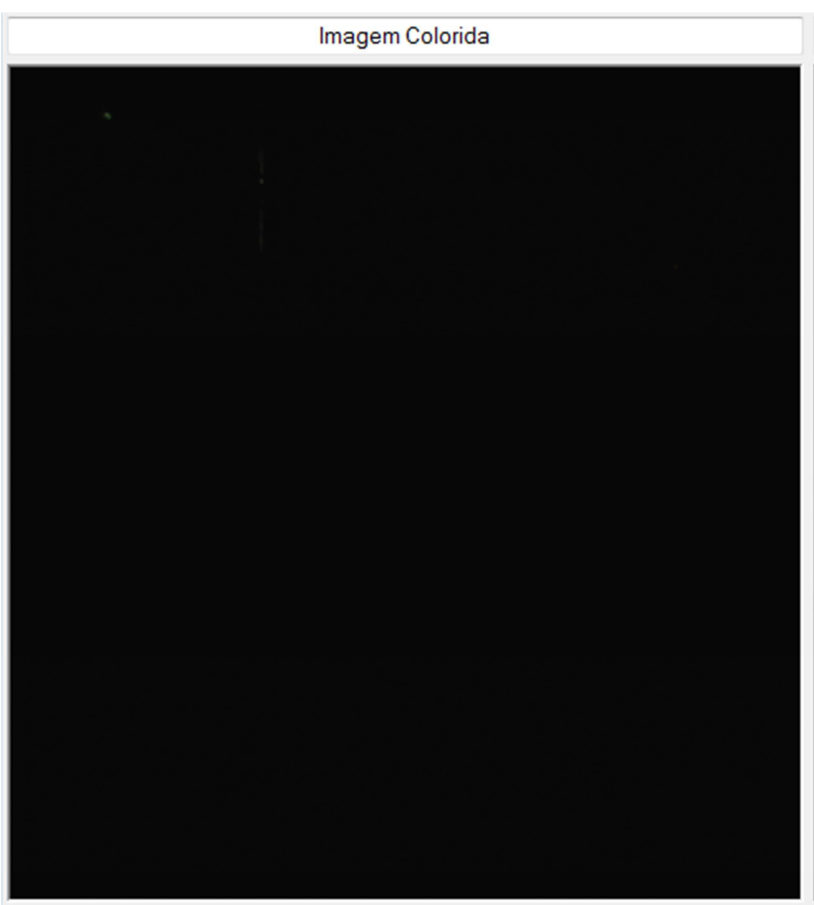

Imagem da Escória

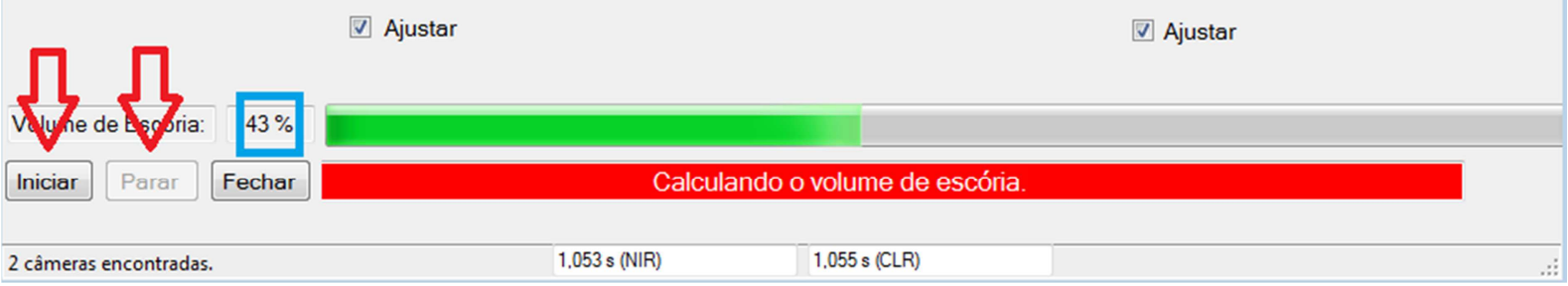

Figure 5. Screenshot from pilot system at the end of skimming process.

On the right side in Figure 5Figure 5, one can see the calculated slag image. The higher the slag, the bigger the pixel value. Visually, maximum pixel value is white and minimum is black. Is also possible to see the final slag index (the number involved by the blue square), $43 \%$, and evaluate skimming performance along with the final image.

The slag index shown on the system screen is a filtered measurement, once the instantaneous index has a high variability, like in Figure 6. In this graphic is possible to understand the slag index variation pattern, which is related to iteration between the rake movement, the slag and the fluid properties of the metal bath, producing waves that temporarily increase the metal surface visibility, and, for consequence, temporarily increasing the slag rate index.

The slag index pattern can also be used for other purposes. In Figure 6 one can conclude that in 17 seconds were performed 2 rakes. Then, this measurement can be used to calculate a productivity index of 0.12 rakes/second. In the future, the system is intended to also perform online evaluation of the skimming process quality and create reports about the parties involved, such as: operators, hot metal quality, equipment performance and process costs. 
$\longrightarrow$ Skimming Process

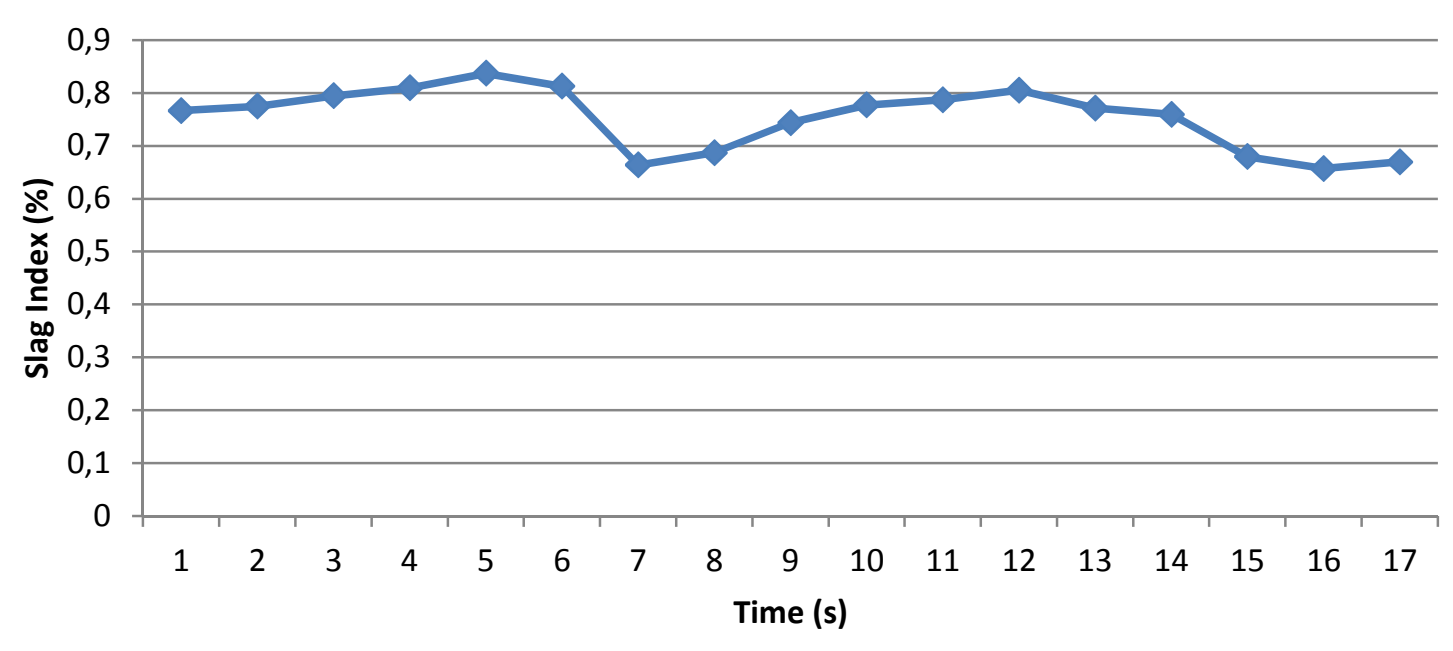

Figure 6. Evolution of instantaneous skimming index.

\section{CONCLUSION}

The current stage of the project shows that is possible to develop a new fully functional robust product, with a low investment profile, using internal human resources and pilot based approach. This was accomplished mainly through the use of solid established tools like Matlab, and, porting the posterior code to a commercial software development tool (Microsoft .Net C\#), generating a hardware independent library set to be used in future artificial vision internal projects. Such a set of libraries can make further development easier to be accomplished.

The use of internal resources is also favored by the previous knowledge of the site and its infrastructure by the team, and also, can generate valuable, configurable and adaptable products, technical skills improvements, interpersonal relations strengthening, and the possibility of reuse of the product on any other compatible site of the group. Another foreseen possibility is to use the gage as a feedback in a total automated loop, with the use of a robot or a revamp of the actual deslagging machine, to make the process even more reliable and profitable.

\section{REFERENCES}

1 F. van der Heijden, "Image Based Measurement Systems", Wiley, 1994.

2 Matlab. The Language of Technical Computing. Available from: <http://www.mathworks.com/products/matlab/>. Acess: 12 mar. 2015.

3 Susstrunk, Sabine, Robert Buckley, and Steve Swen. "Standard RGB color spaces." Color and Imaging Conference. Vol. 1999. No. 1. Society for Imaging Science and Technology, 1999, pp. 127-134.

4 Wen, Che-Yen, and Chun-Ming Chou. "Color Image Models and Its Applications to Document Examination." Forensic Science Journal 3.1.2004.pp. 23- 32.

5 Gonzalez, R.C. and R.E. Woods, "Digital Image Processing" 1992, Reading, Massachusetts: AddisonWesley. 